in detail. It is not, he argues, due to any uniquely pernicious qualities of drug companies since similar trends can be seen in relation to some other types of therapy.

If this additional, temporal dimension had been taken into account, one suspects that Gordon Parker might have placed greater emphasis on one of the factors that he identified as contributing to the current situation: namely that " "depression" is currently modelled as a single entity, varying only in severity' (p. 102). The term 'depression' is thus semantically equivalent nowadays to 'abdominal pain', not to 'appendicitis' or 'peptic ulcer'. If trials of an antacid, say, were undertaken on patients selected for 'abdominal pain' the results obtained would sometimes be favourable, sometimes not. Debate over antacid usefulness would exactly parallel that over the effectiveness of antidepressants.

How did we get into this situation? It seems likely that a lot of the blame can be laid at the door of DSM-III (American Psychiatric Association, 1980), which explicitly aimed for reliability of diagnosis. Unhappily, there was an implicit downside. The state of the art in psychiatry, when DSM-III was under development, was such that reliability could be attained only at the expense of validity. Partly as a consequence of choices that were made then, this problem still remains. It is no good blaming the failings of clinical trials, the machinations of drug companies, the uselessness of antidepressants or reporting bias, for our predicament. The main fault lies in the consequences of a bad choice of diagnostic system, made by our predecessors for what seemed, at the time, good reasons. The remedy must lie primarily in seeing DSM for the hindrance that it is, and one day replacing it with a system that separates the 'peptic ulcers' from the 'appendicitises'.

American Psychiatric Association (1980) Diagnostic and Statistical Manual of Mental Disorders (3rd edn) (DSM-III). Washington, DC: APA.

Healy, D. (2002) The Creation of Psychopharmacology. Cambridge, MA: Harvard University Press.

Parker, G./Anderson I. M. \& Haddad, P. (2003) In debate: Clinical trials of antidepressants are producin meaningless results. British Journal of Psychiatry, $\mathbf{1 8 3}$, 102-104.

C. M. H. Nunn Barfad Beag, Ardfern, Argyll PA3I 8QN, UK

\section{Integrity and bias in academic psychiatry}

The illuminating discussion by Drs Healy and Thase (2003) focuses on the magnitude of the pharmaceutical industry's influence on academic medicine. However, this discussion needs to be taken a step further, and evaluated in relation to patient care. From my perspective, the central question is: 'Does the influence of the pharmaceutical industry on academia result in biased knowledge?' Professionals are charged with serving the best interests of patients/clients. In order to accomplish this, professionals need unbiased knowledge that can lead to an accurate risk-benefit assessment and serve to guide clinical decisions. If available knowledge is biased, decisions will be affected and clients will suffer accordingly. The frequently touted disclosure of potential conflicts of interest in academic publications is a small step in addressing the much more difficult question of whether existing knowledge is biased. Recognising potential bias is an initial step towards assessing and removing it from the collective knowledge used to make decisions in practice. For example, registering clinical trials is an approach to reducing publication bias (Dickersin \& Rennie, 2003). Meta-analysis is an approach to removing bias from expert reviews of the literature (Beaman, 1991), although expert reviews still retain influence in the formulation of some practice guidelines (e.g. American Psychiatric Association, 1997). As the field moves more towards the implementation of evidence-based practice guidelines, the importance of removing bias remains central to providing optimal clinical care. If the extensive financial arrangements between industry and academia resulted in no bias to knowledge, I would probably agree with Dr Thase that no new policies are necessary to 'safeguard our integrity' (p. 390). However a recent systematic review and meta-analysis of evidence bearing on this question found 'strong and consistent evidence... that industry-sponsored research tends to draw pro-industry conclusions' (Bekelman et al, 2003: p. 463). The question now becomes, 'What safeguards should be implemented to remove this bias from the knowledge that guides clinical practice (cf. Bodenheimer, 2000)?' Commitment to our patients' well-being requires that we act from this integrity.

American Psychiatric Association (1997) Practice guidelines for the treatment of patients with schizophrenia. American Journal of Psychiatry, 154, I-63.

Beaman, A. I. (1991) An empirical comparison of metaanalytic and traditional reviews. Personality and Social Psychology Bulletin, I7, 252-257.
Bekelman, J. E., Li, Y. \& Gross, C. P. (2003) Scope and impact of financial conflicts of interest in biomedical research: a systematic review. JAMA, 289, 454-465.

Bodenheimer, T. (2000) Uneasy alliance - clinical investigators and the pharmaceutical industry. New England Journal of Medicine, 342, 1539-1544.

Dickersin, K. \& Rennie, D. (2003) Registering clinical trials. JAMA, 290, 516-523.

(2003) Is academic psychiatry for sale? British Journal of Psychiatry, 182, 388-390.

J. R. Bola School of Social Work, University of Southern California, Los Angeles, CA 90089-04II, USA

\section{Good practice in publication of clinical trial results}

As the name implies, ghostwriting is often hard to detect, so Healy \& Cattell (2003) have made a valuable contribution to our understanding of this important subject by their measurement and thoughtful analysis of the practice. It is also refreshing to see such a balanced account which discusses both the benefits and potential dangers of ghostwriting.

Perhaps their most alarming observation is that the papers sponsored by the manufacturer reported 'universally positive results', which implies the existence of considerable publication bias. Such distortions to the published literature probably exist across all therapeutic areas and have been shown to distort the outcomes of meta-analyses (Tramèr et al, 1997) and therefore to have serious implications for evidence-based medicine (Melander et al, 2003).

Readers may be interested to know that guidelines have recently been published which call on pharmaceutical companies to endeavour to publish results of all clinical trials of marketed products (Wager et al, 2003). The guidelines also provide recommendations to ensure that professional medical (ghost)writers are used appropriately so that their contribution can be beneficial rather than harmful. The Good Publication Practice (GPP) for pharmaceutical companies guidelines have been publicly endorsed by several drug companies and communications agencies. Further details are available at http://www.gpp-guidelines.org.

\section{Declaration of interest}

E.W. is an author of the GPP for pharmaceutical companies guidelines. $\mathrm{He}$ also makes a living as a freelance medical writer, which sometimes involves ghostwriting. 